\title{
Plasma Phosphoproteome and Differential Plasma Phosphoproteins with Opisthorchis Viverrini-Related Cholangiocarcinoma
}

\author{
Kanawut Kotawong ${ }^{1,2}$, Veerachai Thitapakorn ${ }^{1,2}$, Sittiruk Roytrakul ${ }^{3}$, Narumon \\ Phaonakrop $^{3}$, Vithoon Viyanant ${ }^{1,2}$, Kesara Na-Bangchang ${ }^{1,2,4 *}$
}

\begin{abstract}
This study was conducted to investigate the plasma phosphoproteome and differential plasma phosphoproteins in cases of of Opisthorchis viverrini (OV)-related cholangiocarcinoma (CCA). Plasma phosphoproteomes from CCA patients (10) and non-CCA subjects (5 each for healthy subjects and $\mathrm{OV}$ infection) were investigated using gel-based and solution-based LC-MS/MS. Phosphoproteins in plasma samples were enriched and analyzed by LC-MS/MS. STRAP, PANTHER, iPath, and MeV programs were applied for the identification of their functions, signaling and metabolic pathways; and for the discrimination of potential biomarkers in CCA patients and non-CCA subjects, respectively. A total of 90 and 60 plasma phosphoproteins were identified by gel-based and solution-based LC-MS/MS, respectively. Most of the phosphoproteins were cytosol proteins which play roles in several cellular processes, signaling pathways, and metabolic pathways (STRAP, PANTHER, and iPath analysis). The absence of serine/arginine repetitive matrix protein 3 (A6NNA2), tubulin tyrosine ligase-like family, member 6 , and biorientation of chromosomes in cell division protein 1-like (Q8NFC6) in plasma phosphoprotein were identified as potential biomarkers for the differentiation of healthy subjects from patients with CCA and OV infection. To differentiate CCA from OV infection, the absence of both serine/threonine-protein phosphatase $2 \mathrm{~A} 56 \mathrm{kDa}$ regulatory subunit beta isoform and coiled-coil domain-containing protein 126 precursor (Q96EE4) were then applied. A combination of 5 phosphoproteins may new alternative choices for CCA diagnosis.
\end{abstract}

Keywords: Biomarkers - cholangiocarcinoma - LC-MS/MS - phosphoproteomes

Asian Pac J Cancer Prev, 16 (3), 1011-1018

\section{Introduction}

Phosphoproteins, which make up about $30 \%$ of total human proteins, play major roles in cell proliferation, differentiation, division, signaling, and metabolism (Ma et al., 2005; Skeen et al., 2006; Bian et al., 2013; Lien et al., 2013; Liang et al., 2014). Alterations of their phosphorylation and de-phosphorylation activities have been shown to be associated with the pathogenesis of several diseases including diabetes, Alzheimer's disease, multiple myeloma, major depressive disorder, and cancer (Lin et al., 1999; Guha et al., 2008; Ge et al., 2010; Martins-de-Souza et al., 2012; van Abel et al., 2012; Liao et al., 2013). It is also shown that one of the mechanisms of carcinogenesis in cholangiocarcinoma (CCA), the cancer of bile duct, is the alteration of phosphorylated activity resulting from these changes (Kang et al., 2002; Lee et al., 2012; Yonglitthipagon et al., 2012). This type of cancer constitutes a serious health problem with high mortality rate in Asian countries particularly in the northeast region of Thailand. The major risk factor of CCA in Thailand is related to the consumption of liver fluke (Opisthorchis viverrini: $\mathrm{OV}$ ) together with the pre-carcinogen nitrosamines containing fermented food "Pla-Ra" and "Pla-Som" (Thamavit et al., 1978; Mitacek et al., 1999). In non-liver fluke endemic countries, primary sclerosing cholangitis (PSC) is the major risk factor of CCA. The mechanisms underlying the carcinogenesis, development, and progression of these two types of CCA are different (Sripa et al., 2007; Tyson and El-Serag, 2011; Eaton et al., 2013). While the mechanisms involved in non-OV related CCA have been relatively well characterized (Sahani et al., 2003; Burak et al., 2004; Tyson and El-Serag, 2011), those involved in CCA have been limited. Only the secretome of OV-related CCA and plasma of CCA hamsters have been investigated (Srisomsap et al., 2010; Khoontawad

${ }^{I}$ Graduate Program in Bioclinical Sciences, ${ }^{2}$ Center of Excellence in Pharmacology and Molecular Biology of Malaria and Cholangiocarcinoma, Chulabhorn International College of Medicine, ${ }^{4}$ Excellence Center in Pharmacology and Molecular Biology of Malaria and Cholangiocarcinoma, Thammasat University, ${ }^{3}$ Center for Genetic Engineering and Biotechnology (BIOTEC), National Science and Technology Development Agency, Pathumthani, Thailand*For correspondence: kesaratmu@yahoo.com 
et al., 2014). Information on the proteome of CCA plasma particularly phosphoproteins could be exploited for therapeutic, diagnostic, and prognostic applications. Furthermore, it also provides essential basic knowledge at the molecular level especially with regard to cell signaling, proliferation, and differentiation, which would lead to the target discovery of anti-CCA drugs. The proteomics of a number of human cells have been investigated using highthroughput liquid chromatography mass-spectrometry (LC-MS/MS) (Srisomsap et al., 2010; White et al., 2014). The aim of this study was to investigate plasma phosphoproteome and identify potential biomarkers for the diagnosis of patients with OV-related CCA. Serum phosphoproteome profiles have been demonstrated as potential markers for diagnosis and prognosis of pancreatic cancer (Li et al., 2013), esophageal squamous cell carcinoma (Wan et al., 2013), and colorectal cancer (Deng et al., 2013). Here, bioinformatics approaches were applied to analyze the phosphoproteome and the potential biomarker to discriminate CCA patient from OV infected patient and healthy subjects.

\section{Materials and Methods}

Plasma samples and enrichment of plasma phosphoproteins. Plasma samples obtained from 10 CCA patients $(8$ males and 2 females, aged 36-72 years) and 10 non-CCA subjects (5 each for healthy subjects and patients with OV infection); 9 males and 1 female, aged 32-63 years) were kindly provided by Dr. Petcharin Srivatanakul of the National Cancer Institute of Thailand. Ethical approval of the study protocol was obtained from the Ethics Committee of the Ministry of Public Health of Thailand. Written informed consent for study participation was obtained from all subjects prior to the study. The diagnosis of CCA was based on ultrasonography, laboratory [tumor markers (alpha-fetoprotein: AFP, CA19-9) and liver function tests (AST, ALT, ALP)], and histopathological examinations. Alpha-1-antitrypsin (AP1), the candidate biomarker of CCA was also included (Sriwanitchrak et al., 2011). Plasma protein concentrations were determined using Lowry's method, with bovine serum albumin (SIGMA-Aldrich, MO,USA) as a reference standard (Lowry et al., 1951). Protein concentration of each sample was adjusted to a final concentration of $10 \mu \mathrm{g} / \mu \mathrm{l}$ and subjected to phosphoprotein enrichment procedure (Phosphoprotein Enrichment Kit, Pierce, IL, USA). The enriched phosphoprotein was concentrated using $9 \mathrm{kDa}$ cut-off membrane column (Phosphoprotein Enrichment Kit, Pierce, IL, USA) and desalted by gel filtration (Thermo Scientific, Rockford, IL, USA). Protein concentration was determined using Lowry's method, and molecular size was determined using $12.5 \%$ SDS-PAGE (Figure 1A).

\section{Analysis of enriched plasma phosphoproteins by LC-MS/ $M S$}

Enriched plasma phosphoproteins from samples obtained from the CCA patients and non-CCA subjects were investigated using two different techniques, i.e., gel-based and solution-based LC-MS/MS.
Gel-based LC-MS/MS: Enriched plasma phosphoproteins from both groups of subjects were initially separated based on molecular size using $12.5 \%$ SDS-PAGE. The patterns and intensity of separated proteins from plasma of both groups of subjects were generally similar, with difference in intensity only for proteins separated at the molecular size of 14-21 kDa (Figure 1A). The samples from three subjects of each group were therefore, further resolved by two dimensional gel electrophoresis (2DE) using ReadyStrip ${ }^{\mathrm{TM}}$ IPG Strip (Bio-Rad, OR, USA). The prominent up-regulated protein spots were cut and washed with sterile water. Following incubation with $100 \%$ acetonitrile, the spots were left dried at room temperature for $5 \mathrm{~min}$, followed by carbamidomethylation with buffer I ( $10 \mathrm{mM}$ each of dithiothreitol and ammonium bicarbonate) and buffer II (100 mM iodoacetamide and $10 \mathrm{mM}$ ammonium bicarbonate) at room temperature for 1 hour each. The spots were digested with trypsin (10 ng trypsin in 50\% acetonitrile/10 $\mathrm{mM}$ ammonium bicarbonate) for 12 hours, and extracted three times with $50 \%$ acetonitrile. The extracts were pooled, concentrated $\left(40^{\circ} \mathrm{C}\right.$ for 12 hours), and dissolved with $0.1 \%$ formic acid. The digested extracts were injected into a $\mu$-precolumn (Monolithic Trap Column: $200 \mu \mathrm{m}, 5 \mathrm{~mm}$ i.d.,Amsterdam, Netherland) coupled with the Ultimate 3000 LC system (Dionex,Bremen, Germany) and ESI-Ion Trap MS (HCT ultra PTM Discovery System, BrukerDaltonik, Bremen, Germany) with electrospray at a flow rate of $20 \mu \mathrm{l} / \mathrm{min}$. Proteins were separated on a nano-column (Monolithic Nano Column: $100 \mu$ m, 5 mmi.d.,Amsterdam, Netherland) with a solvent gradient mobile phase consisting of solvent A ( $0.1 \%$ formic acid) and solvent B (50\% water, $50 \%$ acetonitrile, and $0.1 \%$ formic acid) running at a flow rate of $1 \mu \mathrm{l} / \mathrm{min}$ with $20 \mathrm{~min}$ run time.

Solution-based LC-MS/MS: Solution-based LC-MS/ MS analysis was performed for the enriched plasma phosphoprotein samples from both groups of subjects using $50 \mu \mathrm{g}$ proteins prepared as previously described for the gel-based LC-MS/MS with modification. The enriched plasma phosphoprotein was treated with buffer I and buffer II for 1 hour each. Buffer I was then added and incubated for an additional 1 hour to inhibit the carbamidomethyl reaction. For digestion, a mixture of 10 ng trypsin in $50 \%$ acetonitrile and $10 \mathrm{mM}$ ammonium bicarbonate was added into the solution and incubated overnight at room temperature. Finally, protein solution was injected into LC-MS/MS.

\section{Analysis of LC-MS/MS data}

The obtained LC-MS/MS data from both methods (gel-based and solution-based LC-MS/MS) were analyzed by using the DeCyder MSTM program (Amersham Bioscience AB, Uppsala, Sweden) and MASCOT (http:// www.matrixscience.com). To exclude co-purified nonphosphoproteins, PhosphoSitePlus was applied for the phosphorylation site analysis (http://www.phosphosite. org). Gene ontology, signaling pathways, and metabolic pathways were analyzed by using STRAP (Vivek Bhatia, Boston University School of Medicine), PANTHER (http://www.pantherdb.org) (Mi et al., 2013), and iPath 
Differential Plasma Phosphoproteomes with Opisthorchis viverrini-Related Cholangiocarcinomas

(http://pathways.embl.de/iPath2.cgi) (Yamada et al., 2011), respectively. To identify the differential up- and down-regulated phosphoproteins, the MeV (Multiple Experiment Viewer) program (http://www.tm4.org) was applied with the application of $\mathrm{K}$ mean/median clustering (KMC) and t-test $(\mathrm{p}<0.05)$.

\section{Data analysis}

Comparison of the clinical laboratory data between samples from CCA patients and non-CCA subjects was performed using Mann-Whitney $U$ test at a statistical significance level of $\alpha=0.05$. The performance of the diagnostic tests (laboratory tests and potential biomarkers) to discriminate between CCA patients and non-CCA subjects included AUC--the area under the curve of the receiver-operating characteristic (ROC) curve for diagnostic accuracy (SPSS version 15, NY, USA). The sensitivity, specificity, positive predictive value (PPV), and negative predictive value (NPV) of potential phosphoproteins were calculated using DAG-STAT (Mackinnon, 2000).

\section{Results}

Subjects and phosphoprotein preparation

The concentrations of the liver enzymes ALP and AST, and AP1 of the samples from CCA patients were significantly higher than those from non-CCA subjects [median (range): 316 (171-1,047) vs 80 (44-98) u/l; 54 (2881) vs 30 (20-45) u/l; and 243 (177-415) vs 138 (116-175) $\mathrm{mg} / \mathrm{L}$, respectively $(\mathrm{p}<0.01)$. Serum protein was however, significantly lower in samples from CCA patients [2.05 (1.2-4.4) vs $2.7(1.3-4.3 \mathrm{mg} / \mathrm{ml}), \mathrm{p}<0.05]$. For AST, ALP, plasma protein, and AP1, the AUCs of the ROCs $(0.889$,

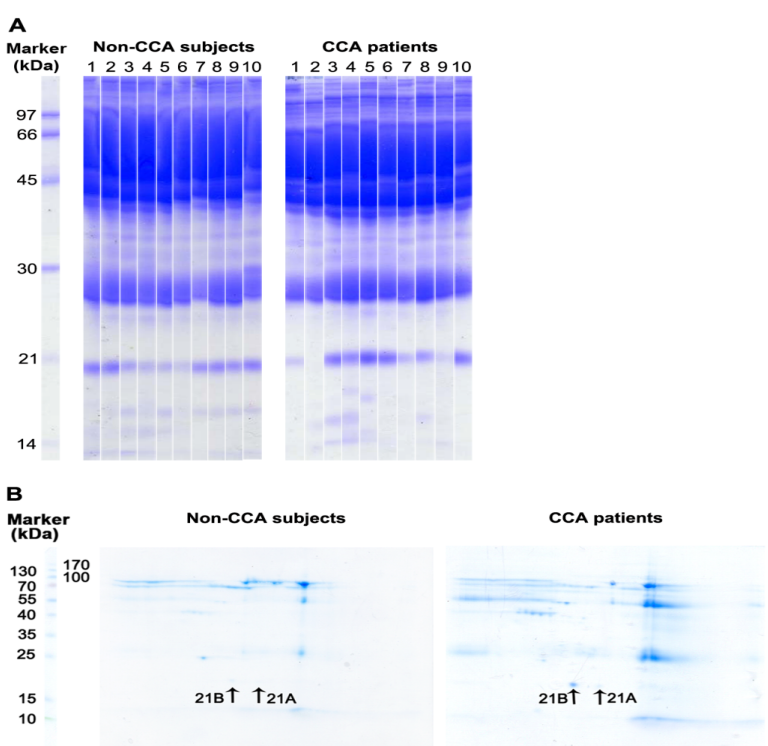

Figure 1. (A) The $12.5 \%$ SDS-PAGE and (B) 2DE of Phosphoproteins of Plasma Samples from CCA Patients and Non-CCA Subjects (10 each)

Table 1. Phosphoproteins and Signaling Pathways Identified by Gel- and Solution-Based LC/MS-MS

\begin{tabular}{|c|c|c|c|c|}
\hline $\begin{array}{l}\text { Pathway (Accession } \\
\text { mapped IDs) }\end{array}$ & Protein & $\begin{array}{l}\text { UniProt } \\
\text { ID }\end{array}$ & Source & $\begin{array}{l}\text { Protein } \\
\text { expression }\end{array}$ \\
\hline \multirow[t]{2}{*}{$\begin{array}{l}\text { PDGF signaling } \\
\text { pathway (P00047) }\end{array}$} & p115 & P98171 & $\begin{array}{l}\text { 21B spot } \\
\text { (gel-based) }\end{array}$ & $\overline{U p}$ \\
\hline & Serine/threonine-protein kinase mTOR & P42345 & In solution & Down \\
\hline \multirow[t]{3}{*}{$\begin{array}{l}\text { FGF signaling pathway } \\
\text { (P00021) }\end{array}$} & $\begin{array}{l}\text { Chain A, Basic Fibroblast Growth Factor Complexed With } \\
\text { Heparin Tetramer Fragment }\end{array}$ & P09038 & $\begin{array}{l}21 \mathrm{~B} \text { spot } \\
\text { (gel-based) }\end{array}$ & Up \\
\hline & $\begin{array}{l}\text { Serine/threonine-protein phosphatase 2A } 56 \mathrm{kDa} \text { regulatory } \\
\text { subunit beta isoform }\end{array}$ & Q15173 & In solution & Down \\
\hline & Serine/threonine-protein kinase mTOR & P42345 & In solution & Down \\
\hline $\begin{array}{l}\text { EGF receptor signaling } \\
\text { pathway (P00018) }\end{array}$ & $\begin{array}{l}\text { Serine/threonine-protein phosphatase 2A } 56 \mathrm{kDa} \text { regulatory } \\
\text { subunit beta isoform }\end{array}$ & Q15173 & In solution & Down \\
\hline $\begin{array}{l}\text { Dopamine receptor } \\
\text { mediated signaling } \\
\text { pathway (P05912) }\end{array}$ & FERM domain-containing protein 6 & Q96NE9 & In solution & Up \\
\hline $\begin{array}{l}\text { Nicotine pharmaco- } \\
\text { dynamics pathway } \\
\text { (P06587) }\end{array}$ & FERM domain-containing protein 6 & Q96NE9 & In solution & Up \\
\hline $\begin{array}{l}\text { Interleukin signaling } \\
\text { pathway (P00036) }\end{array}$ & Serine/threonine-protein kinase mTOR & P42345 & In solution & Down \\
\hline $\begin{array}{l}\text { p53 pathway by glucose } \\
\text { deprivation (P04397) }\end{array}$ & Serine/threonine-protein kinase mTOR & P42345 & In solution & Down \\
\hline $\begin{array}{l}\text { B cell activation } \\
\text { (P00010) }\end{array}$ & Tyrosine-protein kinase BTK & Q06187 & In solution & Down \\
\hline $\begin{array}{l}\text { Hypoxia response } \\
\text { via HIF activation } \\
(\mathrm{P} 00030)\end{array}$ & Serine/threonine-protein kinase mTOR & P42345 & In solution & Down \\
\hline $\begin{array}{l}\text { Wnt signaling pathway } \\
\text { (P00057) }\end{array}$ & $\begin{array}{l}\text { Serine/threonine-protein phosphatase 2A } 56 \mathrm{kDa} \text { regulatory } \\
\text { subunit beta isoform }\end{array}$ & Q15173 & In solution & Down \\
\hline
\end{tabular}


$1,0.985$, and 1 ,respectively) were significantly larger in samples from CCA patients compared with non-CCA subjects.The amounts of enriched plasma phosphoproteins varied markedly in plasma samples from both groups of subjects, but no significant difference was observed [Median (Range): 2.1 (1.2-4.4) and $2.5(1.3-4.3) \mathrm{mg} / \mathrm{ml}$ for CCA and non-CCA subjects, respectively] $(\mathrm{p}=0.307)$.

Analysis of plasma phosphoproteome by gel-based LC$M S / M S$

A total of 300 phosphoprotein spots were resolved (2DE), with two prominent $21 \mathrm{kDa}$ up-regulated spots, namely 21A and 21B (Figure 1B). Results of the LC-MS/ MS analysis revealed 98 and 64 identified proteins from the $21 \mathrm{~A}$ and $21 \mathrm{~B}$ spots. Analysis of the phosphorylation sites by PhosphoSitePlus identified 47 (47.9\%) and 43 (67.2\%) of the $21 \mathrm{~A}$ and $21 \mathrm{~B}$ spots as phosphoproteins, respectively. For the $21 \mathrm{~A}$ spot, the number of phosphoproteins with serine-, threonine-, and tyrosine-phosphorylated sites were 40,32 , and 37 , respectively. For the $21 \mathrm{~B}$ spot, the number of phosphoproteins with serine-, threonine-, and tyrosinephosphorylated sites were 36,34 , and 38, respectively.

Based on the $\mathrm{MeV}$ analysis results, cluster No.1 (out of the default 10 clusters) of the $21 \mathrm{~A}$ spot contained five up-regulated phosphoproteins. These proteins were identified as trafficking protein particle complex subunit 5 (Q8IUR), tumor necrosis factor ligand superfamily member 9 (P41273), peripheral clock protein 2, MFS-type transporter C6orf192 (Q6NT16), and GREB1 protein isoform a (Q4ZG55) (Figure 2A). For the 21B spot, the three up-regulated phosphoproteins in cluster No. 4 were identified as p115 (P98171), basic fibroblast growth factor complexed with heparin tetramer fragment (P09038), and crystal structure of human co-chaperone protein Hscb
(Q8IWL3) (Figure 2B). Further analysis by the $\mathrm{MeV}$ program and statistical analysis by t-test suggested higher amount of the trafficking protein particle complex subunit 5 from the 21A spot in plasma of the CCA compared with non-CCA groups (Figure 3A). For the 21B spot, the amounts of p115 and in addition, hydrocephalus-inducing protein homolog isoform A were significantly higher in the CCA group (Figure 3B). Confirmation by KMC combined with t-test suggested only the trafficking protein particle complex subunit 5 and p115 as potential biomarkers for CCA. It is of interest to further investigate the involvement of these two proteins in cholangiocarcinogenesis in CCA cells. Results of PANTHER analysis also supported this finding, showing the possible association of these two up-regulated phosphoproteins (p115 and chain A basic fibroblast growth factor complexed with heparin tetramer fragment) with cholangiocarcinogenesis through cell signaling pathways (Table 1).

Analysis of phosphoproteome by solution-based LC-MS/ $M S$

A total of 131 proteins were obtained from the LCMS/MS analysis by DeCyder ${ }^{\mathrm{TM}}$ and MASCOT. Sixty $(45.8 \%)$ proteins were identified as phosphoproteins with serine- $(n=52)$, threonine- $(n=39)$, and tyrosine- $(n=49)$ phosphorylation. Results of the MeV-KMC analysis showed similar patterns of plasma phosphoproteinsin CCA and non-CCA subjects (Figure 2C). Using average peak heights of all plasma samples in each group as a differential criterion, 31 and 29 phosphoproteins were classified as the up-and down-regulated phosphoproteins, respectively. Results of the gene ontology analysis revealed that most of these up- and down-regulated phosphoproteins were classified into three main groups based on functions as

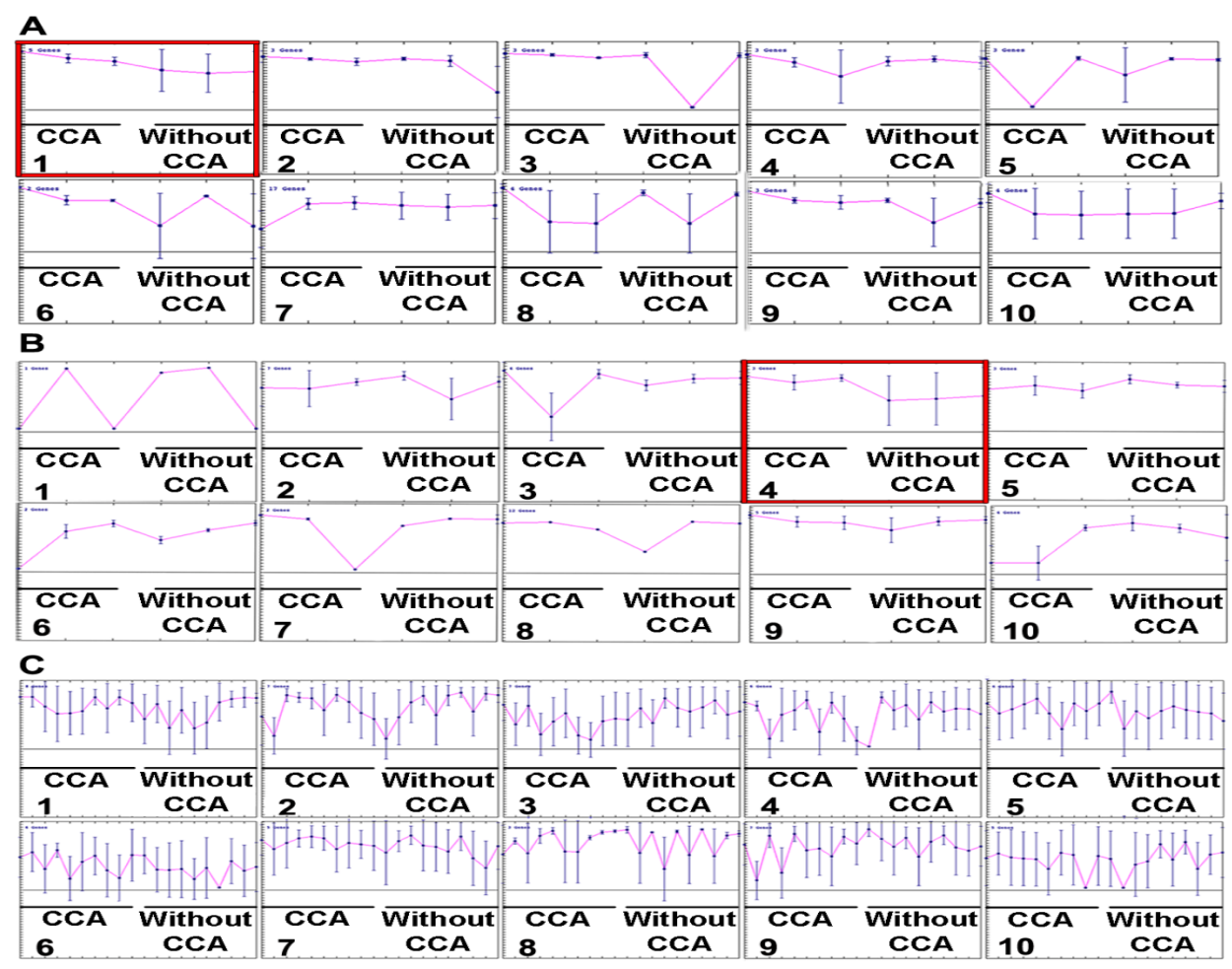

Figure 2. Clustering of Phosphoproteins by MeV-KMC (A) 21A spot (B) 21B Spot, and (C) Solution-based LCMS/MS. Each spot with error bar represents each subject and the number indicates the cluster number 
those involved in $i$ ) biological processes (predominantly regulation process), $i$ i) cellular components (predominantly cytoplasm), and iii) molecular function (predominantly binding proteins). The PANTHER analysis identified four possible phosphoproteins in cell signaling pathways, one up-regulated FERM domain-containing protein 6 , and three down-regulated phosphoproteins (serine/threonineprotein phosphatase $2 \mathrm{~A} 56 \mathrm{kDa}$ regulatory subunit beta isoform, serine/threonine-protein kinase mTOR, and tyrosine-protein kinase BTK) (Table 1). The roles of these phosphoproteins in cholangiocarcinogenesis remain to be elucidated. Results of the iPath analysis identified two phosphoproteins in metabolic pathways, i.e., V-type proton ATPase subunit C 1 of oxidative phosphorylation (up-regulated), and delta-1-pyrroline-5-carboxylate synthase isoform 1 of arginine and proline metabolism

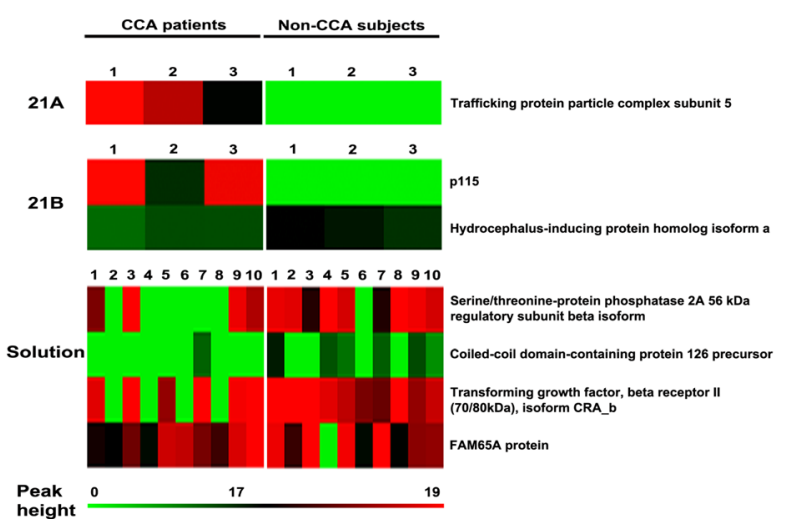

Figure 3. Heatmap of MeV-t-test for Phosphoproteins with Significant Difference in Expression. (A) 21 A spot, (B) 21B spot, and (C) solution-based LC-MS/MS. The color indicates the peak height from low (green) to high (red) (down-regulated). None of the 60 phosphoproteins was identified as proteins involved in cell regulatory pathways and biosynthesis pathways of secondary metabolites.

\section{Identification of plasma phosphoproteins as potential biomarkers of cholangiocarcinoma}

Out of the sixty identified phosphoproteins by solutionbased LC-MS/MS, the MeV-t-test results identified only 4 phosphoproteins with marked difference in amounts (peak heights) between plasma of CCA patients and nonCCA subjects. These included serine/threonine-protein phosphatase 2A $56 \mathrm{kDa}$ regulatory subunit beta isoform (Q15173), coiled-coil domain-containing protein 126 precursor (Q96EE4), transforming growth factor beta receptor II $(70 / 80 \mathrm{kDa})$ isoform CRA_b, and FAM65A protein (Figure 3C). The sensitivity, specificity, PPV, and NPV of these proteins as biomarkers for CCA are summarized in Table 2.

In order to identify potential biomarkers for $\mathrm{OV}$ infection, the average peak heights from solution-based LC-MS/MS analysis of plasma from CCA patient $(n=10)$, healthy subjects $(n=5)$ and those with OV infection $(n=5)$ was compared. Six patterns (A, B, C, D, E, and F) of peak heights were observed (Figure 4). However, only pattern $\mathrm{D}$ (consisting of 2 phosphoproteins) and $\mathrm{F}$ (consisting of 8 phosphoproteins) with a marked difference in average peak heights could discriminate individuals with $\mathrm{OV}$ infection from those with CCA (OV-related). Finally, results of both $\mathrm{MeV}$-t-test and average peak height analysis identified only two phosphoproteins [coiledcoil domain-containing protein 126 precursor (Q96EE4) and serine/threonine-protein phosphatase $2 \mathrm{~A} 56 \mathrm{kDa}$ regulatory subunit beta isoform (Q15173)] as potential

Table 2. Solution-Based LC-MS/MS Analysis of Potential Phosphoproteins

\begin{tabular}{|l|l|c|c|c|c|c|}
\hline No & Name & UniPort ID & Sensitivity (\%) & Specificity (\%) & PPV & NPV \\
\hline 1 & Coiled-coil domain-containing protein 126 precursor & Q96EE4 & 90 & 60 & 0.69 & 0.86 \\
\hline 2 & FAM65A protein & - & 90 & 30 & 0.56 & 0.75 \\
\hline 3 & $\begin{array}{l}\text { Serine/threonine-protein phosphatase 2A 56 kDa } \\
\text { regulatory subunit beta isoform }\end{array}$ & Q15173 & 60 & 90 & 0.86 & 0.96 \\
\hline 4 & $\begin{array}{l}\text { Transforming growth factor, beta receptor II (70/80 } \\
\text { kDa), isoform CRA_b }\end{array}$ & - & 40 & 80 & 0.67 & 0.57 \\
\hline
\end{tabular}

Table 3. Potential Biomarkers for CCA Diagnosis

\begin{tabular}{|l|l|l|l|}
\hline Test & Solution-based phosphoproteins & 21A spot & 21B spot \\
\hline MeV-KMC & Not significant & $\begin{array}{l}\text { trafficking protein } \\
\text { particle complex } \\
\text { subunit 5 (Q8IUR0) }\end{array}$ & p115 (P98171) \\
\hline MeV-t-test & $\begin{array}{l}\text { (1) serine/threonine-protein phosphatase 2A 56 } \\
\text { kDa regulatory subunit beta isoform (Q15173) } \\
\text { (2) coiled-coil domain-containing protein 126 } \\
\text { precursor (Q96EE4) } \\
\text { (3) transforming growth factor beta receptor II } \\
\text { (70/80kDa) isoform CRA_b } \\
\text { (4) FAM65A protein }\end{array}$ & $\begin{array}{l}\text { trafficking protein } \\
\text { subunit 5(Q8IUR0) }\end{array}$ & $\begin{array}{l}\text { (1) p115(P98171) } \\
\text { ing protein homolog } \\
\text { isoform A }\end{array}$ \\
\hline $\begin{array}{l}\text { Exclusive phosphopro- } \\
\text { tein was presented only } \\
\text { in healthy subjects }\end{array}$ & $\begin{array}{l}\text { 1) biorientation of chromosomes in cell division } \\
\text { protein 1-like } \\
\text { (2) serine/arginine repetitive matrix protein 3 } \\
\text { (3) Tubulin tyrosine ligase-like family, member }\end{array}$ & $\begin{array}{l}\text { trafficking protein } \\
\text { particle complex } \\
\text { subunit 5(Q8IUR0) }\end{array}$ & p115 (P98171) \\
\hline
\end{tabular}




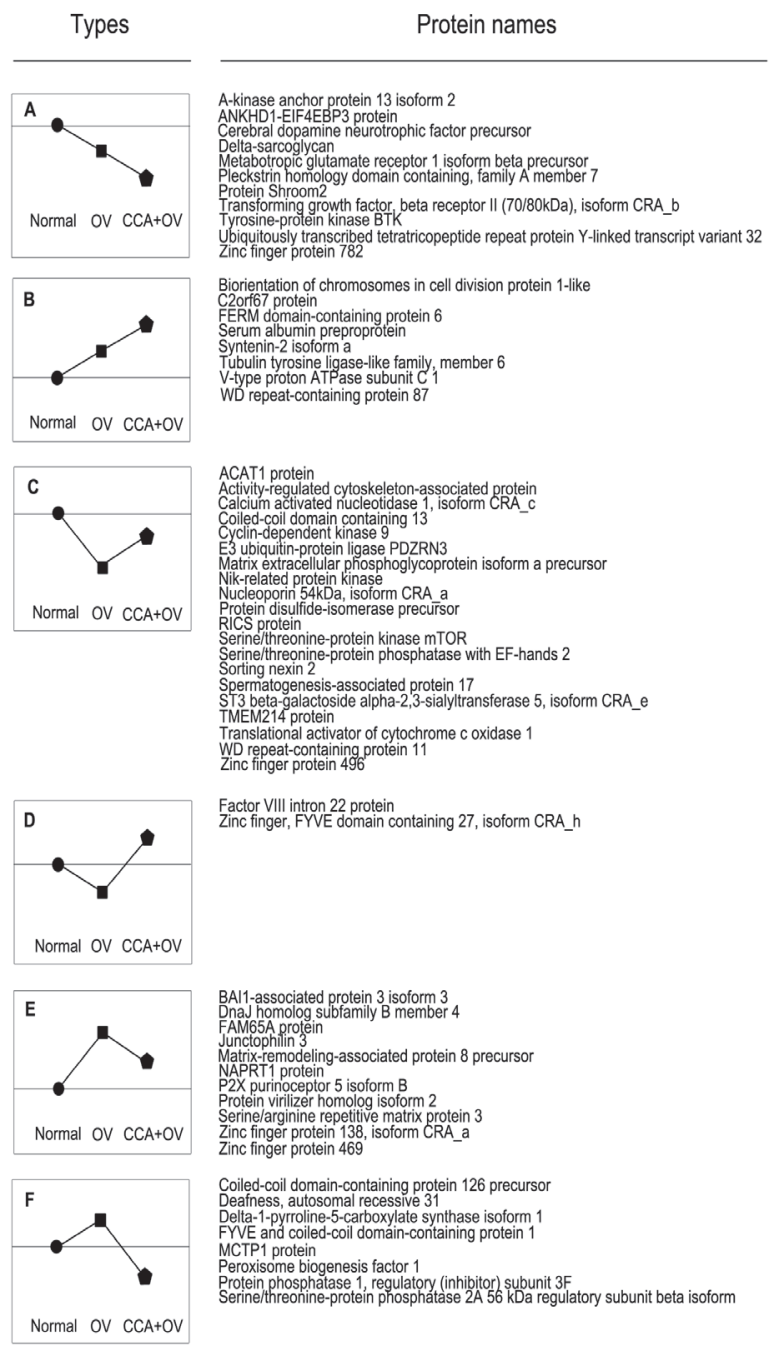

Figure 4. Classification of CCA Patients (CCA), 0. Viverrini Infected Patients $(\mathrm{OV})$, and Healthy Subjects (HS) Based on the Averaged Peak Heights

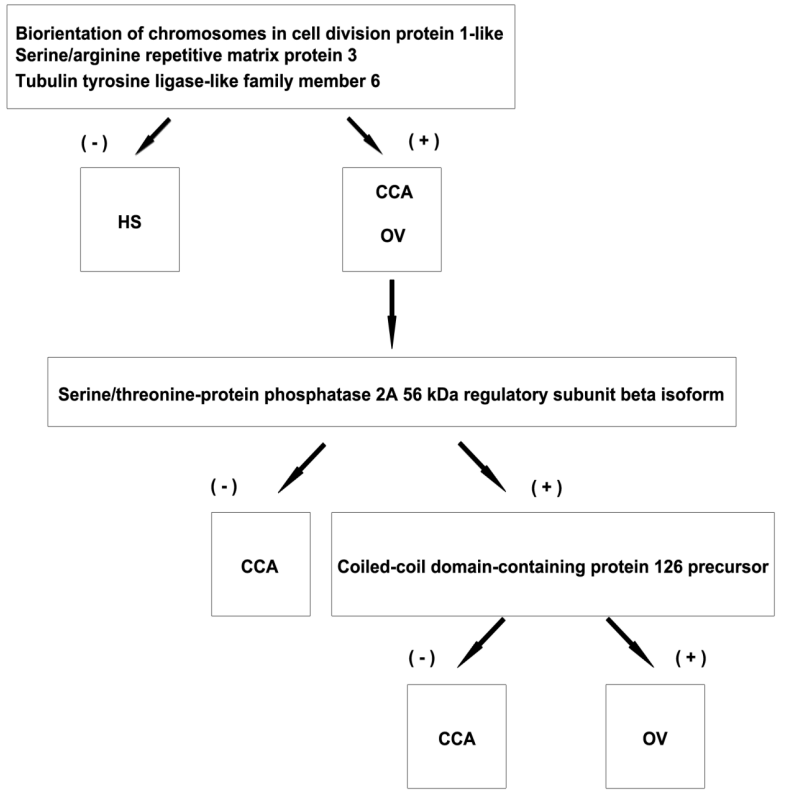

Figure 5. The Proposed Diagnostic Scheme for CCA. The symbol (-) and (+) indicate the absence and presence of plasma phosphoprotein, respectively. CCA (CCA patients, OV /O. viverrini infected patients), and HS (healthy subjects) biomarkers for discrimination between OV infected subjects and CCA (OV-related) patients. In addition, the three phosphoproteins specifically expressed in CCA and $\mathrm{OV}$ were serine/arginine repetitive matrix protein 3 (A6NNA2), tubulin tyrosine ligase-like family member 6 , and biorientation of chromosomes in cell division protein 1-like (Q8NFC6).

\section{Discussion}

Almost all laboratory tests and candidate biomarkers of CCA (plasma protein, AST, ALT, ALP, AP1, and CA 19-9) except AFP were shown in this study to be useful screening tests for CCA. In our previous study, a battery tests consisting of these tests were shown to produce a reliable diagnostic performance for CCA (Sriwanitchrak et al., 2011). However, these liver enzymes and tumor markers when used independently and separately have been reported to be relatively non-specific to CCA as the levels also increased in other diseases (Goni et al., 2013; Kunutsor et al., 2013; Zhang et al., 2013). Unexpectedly, such increase in the levels of these enzymes and tumor markers were not well correlated with the levels of plasma proteins. This suggests the possibility of other existing unidentified proteins as potential biomarkers of CCA.

Results of proteome analysis by gel-based and solutionbased LC-MS/MS were inconsistent in identifying phosphoproteome and differential protein expression in CCA patients and non-CCA subjects (Table 3). For the gel-based LC-MS/MS analysis, up to 98 proteins were identified from a single plasma phosphoprotein spot. This high number of identified proteins could be due to their similarity of pI and molecular weight, as well as the resolution of SDS-PAGE. Using the MeV program with the application of KMC and t-test, two phosphoproteins from the 21A spot (trafficking protein particle complex subunit 5) and 21B (p115) were significantly up-regulated in CCA patients. The trafficking protein particle complex subunit 5 plays a role in vesicular transport from endoplasmic reticulum to Golgi, while the p115 plays a role in PDGF receptor signaling pathway (Sacher et al., 1998; Bai et al., 2011). Up-regulation of the first could contribute to the increase in the activity of the CCA cell in the transportation of the secreted and/or membrane proteins, or serve as an alternative protein trafficking pathway in CCA.

On the other hand, the up-regulation of p115 protein could enhance a signaling activity in the PI3K, JAK/STAT, and MEK/ARK pathways in CCA cells. With regard to the solution-based LC-MS/MS analysis, FAM65A protein was significantly up-regulated, while coiled-coil domain containing protein 126 precursor (Q96EE4), serine/ threonine-protein phosphatase $2 \mathrm{~A} 56 \mathrm{kDa}$ regulatory subunit beta isoform (Q15173) and transforming growth factor beta receptor II $(70 / 80 \mathrm{kDa})$ isoform CRA_b was down-regulated in CCA. The serine/threonine-protein phosphatase $2 \mathrm{~A} 56 \mathrm{kDa}$ regulatory subunit beta isoform was identified as molecules involved in EGF and FGF receptor, as well as Wnt signaling pathways (Letourneux et al., 2006; Eichhorn et al., 2009; Rodgers et al., 2011). The observation of down regulation of these plasma 
phosphoproteins in CCA patients could imply that the chaperon protein was sufficiently expressed in CCA cell, allowing the cell to escape from apoptosis. Elucidation of the involvement of phosphoproteins in the complete network of signaling pathways in CCA was not possible in this study due to the limitation in the use of plasma as study sample. Approximately 4,000 phosphoproteins have been curated in the PhosphoSitePlus database (Yang et al., 2008); mostly intracellular proteins (Wang et al., 2011; Martins-de-Souza et al., 2012), with only small proportion of secreting and excreting phosphoproteins. It is estimated that about $30 \%$ of human proteins are phosphorylated proteins, but the exact proportion of the total plasma phosphoproteins remains unclear. Results of the present study showed that only a small amount (2-4\%) of enriched phosphoproteins was obtained from plasma of both groups of subjects.

Since almost all CCA cases in Thailand are related to $\mathrm{OV}$ infection, the present study also investigated the biomarkers that would potentially discriminate CCA (OV-related) from OV infected patients. The analysis of patterns of average peak heights together with the MeVt-test identified 10 phosphoproteins (peak height patterns $\mathrm{D}$ and F) and 4 phosphoproteins [FAM65A protein, coiled-coil domain containing protein 126 precursor (Q96EE4), serine/threonine-protein phosphatase 2A $56 \mathrm{kDa}$ regulatory subunit beta isoform (Q15173), and transforming growth factor beta receptor II $(70 / 80 \mathrm{kDa})$ isoform CRA_b], respectively. Interestingly, both methods identified serine/threonine-protein phosphatase 2A $56 \mathrm{kDa}$ regulatory subunit beta isoform and coiled-coil domain containing protein 126 precursor phosphoproteins as potential biomarkers of CCA.

In conclusion, the present study identified several potential biomarkers of CCA (OV-related) and OV infection (Table 3). Serine/arginine repetitive matrix protein 3 (A6NNA2), tubulin tyrosine ligase-like family, member 6 , and biorientation of chromosomes in cell division protein 1-like (Q8NFC6) were identified as potential biomarkers for the screening of healthy subjects from CCA and OV infected patients (Figure 5). On the other hand, coiled-coil domain-containing protein 126 precursor (Q96EE4) and serine/threonine-protein phosphatase 2A $56 \mathrm{kDa}$ regulatory subunit beta isoform (Q15173) were identified as potential biomarkers for the differential diagnosis of CCA (OV-related) and OV infected patients. Additionally, the down-regulated serine/ threonine-protein kinase mTOR could be a potential drug target for CCA (Moolthiya et al., 2014). Validation of diagnostic performance of these biomarkers in a large number of patients (CCA and OV infected) is required before a large scale clinical application of these proteins as a screening tool for CCA is to be adopted. Nevertheless, application of these phosphoproteins as diagnostic or prognostic biomarkers of CCA and OV may be limited by their relatively high cost and test complexity. It is likely that a combination of biomarkers together with liver function tests as a battery of tests, rather than a single test, would be a more reliable screening tool for CCA (Malaguarnera et al., 2013).

\section{Acknowledgements}

This study was supported by the National Research University Project (NRU) of Thailand Office of Higher Education Commission, Ministry of Education of Thailand and Thammasat University (Excellence Center in Pharmacology and Molecular Biology of Malaria and Cholangiocarcinoma).

\section{References}

Bai Y, Luo Y, Liu S, et al (2011). PRL-1 protein promotes ERK1/2 and RhoA protein activation through a noncanonical interaction with the Src homology 3 domain of p115 Rho GTPase-activating protein. J Biol Chem, 286, 42316-24.

Bian S, Sun X, Bai A, et al (2013). P2X7 integrates PI3K/AKT and AMPK-PRAS40-mTOR signaling pathways to mediate tumor cell death. PLoS One, 8, 60184.

Burak K, Angulo P, Pasha TM, et al (2004). Incidence and risk factors for cholangiocarcinoma in primary sclerosing cholangitis. Am J Gastroenterol, 99, 523-6.

Deng BG, Yao JH, Liu QY, et al (2013). Comparative serum proteomic analysis of serum diagnosis proteins of colorectal cancer based on magnetic bead separation and Maldi-tof mass spectrometry. Asian Pac J Cancer Prev, 14, 6069-75.

Eaton JE, Talwalkar JA, Lazaridis KN, et al (2013). Pathogenesis of primary sclerosing cholangitis and advances in diagnosis and management. Gastroenterology, 145, 521-36.

Eichhorn PJ, Creyghton MP, Bernards R (2009). Protein phosphatase $2 \mathrm{~A}$ regulatory subunits and cancer. Biochim Biophys Acta, 1795, 1-15.

Ge F, Xiao CL, Yin XF, et al (2010). Phosphoproteomic analysis of primary human multiple myeloma cells. J Proteomics, 73, 1381-90.

Goni BW, Yusuph H, Mustapha SK, et al (2013). Hepatic transaminase and alkaline phosphatase enzyme levels in HIV/HBV co-infected and HIV mono-infected patients in Maiduguri, Nigeria. Niger J Clin Pract, 16, 530-4.

Guha U, Chaerkady R, Marimuthu A, et al (2008). Comparisons of tyrosine phosphorylated proteins in cells expressing lung cancer-specific alleles of EGFR and KRAS. Proc Natl Acad Sci USA, 105, 14112-7.

Kang YK, Kim WH, Jang JJ (2002). Expression of G1-S modulators (p53, p16, p27, cyclin D1, Rb) and Smad4/Dpc4 in intrahepatic cholangiocarcinoma. Hum Pathol, 33, 877-83.

Khoontawad J, Hongsrichan N, Chamgramol Y, et al (2014). Increase of exostosin 1 in plasma as a potential biomarker for opisthorchiasis-associated cholangiocarcinoma. Tumour Biol, 35, 1029-39.

Kunutsor SK, Apekey TA, Walley J (2013). Liver aminotransferases and risk of incident type 2 diabetes: a systematic review and meta-analysis. Am J Epidemiol, 178, 159-71.

Lee D, Do IG, Choi K, et al (2012). The expression of phosphoAKT1 and phospho-MTOR is associated with a favorable prognosis independent of PTEN expression in intrahepatic cholangiocarcinomas. Mod Pathol, 25, 131-9.

Letourneux C, Rocher G, Porteu F (2006). B56-containing PP2A dephosphorylate ERK and their activity is controlled by the early gene IEX-1 and ERK. EMBO J, 25, 727-38.

Li P, Yang J, Ma QY, et al (2013). Biomarkers screening between preoperative and postoperative patients in pancreatic cancer. Asian Pac J Cancer Prev, 14, 4161-5.

Liang D, Zeng Q, Xu Z, et al (2014). BAFF activates Erk1/2 promoting cell proliferation and survival by $\mathrm{Ca} 2+-\mathrm{CaMKII-}$ 
Kanawut Kotawong et al

dependent inhibition of PP2A in normal and neoplastic B-lymphoid cells. Biochem Pharmacol, 87, 332-43.

Liao MH, Xiang YC, Huang JY, et al (2013). The disturbance of hippocampal CaMKII/PKA/PKC phosphorylation in early experimental diabetes mellitus. CNS Neurosci Ther, 19, 329-36.

Lien SC, Chang SF, Lee PL, et al (2013). Mechanical regulation of cancer cell apoptosis and autophagy: roles of bone morphogenetic protein receptor, Smad1/5, and p38 MAPK. Biochim Biophys Acta, 1833, 3124-33.

Lin J, Adam RM, Santiestevan E, et al (1999). The phosphatidylinositol 3'-kinase pathway is a dominant growth factor-activated cell survival pathway in $\mathrm{LNCaP}$ human prostate carcinoma cells. Cancer Res, 59, 2891-7.

Lowry OH, Rosebrough NJ, Farr AL, et al (1951). Protein measurement with the Folin phenol reagent. J Biol Chem, 193, 265-75.

Ma L, Chen Z, Erdjument-Bromage H, et al (2005). Phosphorylation and functional inactivation of TSC2 by Erk implications for tuberous sclerosis and cancer pathogenesis. Cell, 121, 179-93.

Mackinnon A (2000). A spreadsheet for the calculation of comprehensive statistics for the assessment of diagnostic tests and inter-rater agreement. Comput Biol Med, 30, 127-34.

Malaguarnera G, Paladina I, Giordano M, et al (2013). Serum markers of intrahepatic cholangiocarcinoma. Dis Markers, 34, 219-28.

Martins-de-Souza D, Guest PC, Vanattou-Saifoudine N, et al (2012). Phosphoproteomic differences in major depressive disorder postmortem brains indicate effects on synaptic function. Eur Arch Psychiatry Clin Neurosci, 262, 657-66.

Mi H, Muruganujan A, Casagrande JT, et al (2013). Large-scale gene function analysis with the PANTHER classification system. Nat Protoc, 8, 1551-66.

Mitacek EJ, Brunnemann KD, Suttajit M, et al (1999). Exposure to N-nitroso compounds in a population of high liver cancer regions in Thailand: volatile nitrosamine (VNA) levels in Thai food. Food Chem Toxicol, 37, 297-305.

Moolthiya P, Tohtong R, Keeratichamroen S, et al (2014). Role of mTOR inhibitor in cholangiocarcinoma cell progression. Oncol Lett, 7, 854-60.

Rodgers JT, Vogel RO, Puigserver P (2011). Clk2 and B56beta mediate insulin-regulated assembly of the PP2A phosphatase holoenzyme complex on Akt. Mol Cell, 41, 471-9.

Sacher M, Jiang Y, Barrowman J, et al (1998). TRAPP, a highly conserved novel complex on the cis-Golgi that mediates vesicle docking and fusion. EMBO J, 17, 2494-503.

Sahani D, Prasad SR, Tannabe KK, et al (2003). Thorotrastinduced cholangiocarcinoma: case report. Abdom Imaging, 28, 72-4.

Skeen JE, Bhaskar PT, Chen CC, et al (2006). Akt deficiency impairs normal cell proliferation and suppresses oncogenesis in a p53-independent and mTORC1-dependent manner. Cancer Cell, 10, 269-80.

Sripa B, Kaewkes S, Sithithaworn P, et al (2007). Liver fluke induces cholangiocarcinoma. PLoS Med, 4, 201.

Srisomsap C, Sawangareetrakul P, Subhasitanont P, et al (2010). Proteomic studies of cholangiocarcinoma and hepatocellular carcinoma cell secretomes. J Biomed Biotechnol, 2010, 437143.

Sriwanitchrak P, Viyanant V, Chaijaroenkul W, et al (2011). Proteomics analysis and evaluation of biomarkers for detection of cholangiocarcinoma. Asian Pac J Cancer Prev, 12, 1503-10.

Thamavit W,Bhamarapravati N, Sahaphong S, et al (1978). Effects of dimethylnitrosamine on induction of cholangiocarcinoma in Opisthorchis viverrini-infected Syrian golden hamsters. Cancer Res, 38, 4634-9.

Tyson GL, El-Serag HB (2011). Risk factors for cholangiocarcinoma. Hepatology, 54, 173-84.

van Abel D, Abdulhamid O, Scheper W, et al (2012). STOX1A induces phosphorylation of tau proteins at epitopes hyperphosphorylated in Alzheimer's disease. Neurosci Lett, 528, 104-9.

Wan QL, Hou XS, Zhao G (2013). Utility of serum peptidome patterns of esophageal squamous cell carcinoma patients for comprehensive treatment. Asian Pac J Cancer Prev, 14, 2919-23.

Wang X, Stewart PA, Cao Q, et al (2011). Characterization of the phosphoproteome in androgen-repressed human prostate cancer cells by Fourier transform ion cyclotron resonance mass spectrometry. J Proteome Res, 10, 3920-8.

White NM, Masui O, Desouza LV, et al (2014). Quantitative proteomic analysis reveals potential diagnostic markers and pathways involved in pathogenesis of renal cell carcinoma. Oncotarget.

Yamada T, Letunic I, Okuda S, et al (2011). iPath2.0: interactive pathway explorer. Nucleic Acids Res, 39, 412-5.

Yang CY, Chang CH, Yu YL, et al (2008). PhosphoPOINT: a comprehensive human kinase interactome and phosphoprotein database. Bioinformatics, 24, 14-20.

Yonglitthipagon P, Pairojkul C, Chamgramol Y, et al (2012). Prognostic significance of peroxiredoxin 1 and ezrin-radixinmoesin-binding phosphoprotein 50 in cholangiocarcinoma. Hum Pathol, 43, 1719-30.

Zhang S, Chen Y, Zhu Z, et al (2013). Differential expression of carbohydrate antigen 19-9 in human colorectal cancer: A comparison with colon and rectal cancers. Mol Clin Oncol, 1, 1072-8. 